\title{
Myocardial Ischemia Caused by Subepicardial Hematoma
}

\author{
Philippe Grieshaber $^{1}$ Holger Nef ${ }^{2}$ Andreas Böning ${ }^{1}$ Bernd Niemann ${ }^{1}$ \\ ${ }^{1}$ Department of Adult and Pediatric Cardiovascular Surgery, \\ University Hospital of Giessen, Giessen, Germany \\ 2 Department of Cardiology and Angiology, University Hospital of \\ Giessen, Giessen, Germany

\begin{abstract}
Address for correspondence Philippe Grieshaber, MD, Department of Adult and Pediatric Cardiovascular Surgery, University Hospital of Giessen, Rudolf-Buchheim-Str. 7, Giessen 35392, Germany
\end{abstract} \\ (e-mail: philippe.grieshaber@chiru.med.uni-giessen.de).
}

Thorac Cardiovasc Surg Rep 2017;6:e5-e9.

\begin{abstract}
Keywords

- complication

- myocardial ischemia

- hematoma

- coronary artery bypass grafting

- CABG

Background Bleeding from bypass anastomosis leakage occurs early after coronary artery bypass grafting. Later, once the anastomosis is covered by intima, spontaneous bleeding is unlikely.

Case Description A 63-year-old male patient developed a pseudoaneurysm-like, subepicardial late-term bleeding resulting in a hematoma that compromised coronary artery flow by increasing extracoronary pressure. This resulted in severe angina pectoris (Canadian Cardiovascular Society IV) and myocardial ischemia within the affected area. After surgical removal of the hematoma and repair of the anastomosis, the patient's symptoms disappeared and no signs of myocardial ischemia were present. Conclusion Surgical removal is an efficient therapy for subepicardial hematoma inducing myocardial ischemia.
\end{abstract}

\section{Introduction}

Anastomosis-related complications after coronary artery bypass grafting (CABG) usually comprise anastomosis narrowing and leakage. Narrowing of an anastomosis can impair bypass function and run-off into the target vessel and is described in as many as $34 \%$ of patients undergoing reangiography due to symptomatic graft failure up to 1 year after CABG. ${ }^{1}$ Anastomosis leakage occurs in 2 to $6 \%$ of CABG procedures, manifests in the immediate perioperative period, and results in acute bleeding, pericardial tamponade, and the need for re-exploration. ${ }^{2}$ The healing process of coronary anastomoses, which includes formation of a neointima and the covering by an epicardial layer, is completed after 30 to 50 days. ${ }^{3}$ Thereafter, spontaneous bleeding from the anastomosis is unlikely; should it occur, however, it would result in a subepicardial hematoma. Formation of a subepicardial hematoma following perforation of a bypass graft during percutaneous coronary intervention of the graft has been described in two cases. ${ }^{4,5}$ Spontaneous subepicardial hematoma due to delayed anastomosis leakage has not been described thus far. Here, we present a case of the formation of an anastomosis-related subepicardial hematoma 7 weeks after CABG that compromised the downstream coronary artery and resulted in myocardial ischemia and acute coronary syndrome.

\section{Case Description}

A 63-year-old male patient underwent aortic valve replacement with a mechanical valve prosthesis (ATS Medical, Open Pivot $29 \mathrm{~mm}$ ) for severe aortic regurgitation and CABG (left internal mammary artery [LIMA] to obtuse marginal branch 2 (OM2). Total postoperative pericardial and pleural drainage volume was $350 \mathrm{~mL}$. Due to intermittent atrioventricular block III postoperatively, a permanent dual chamber pacemaker was implanted on postoperative day (POD) 5 . After an uneventful postoperative period, the patient was discharged to cardiovascular rehabilitation on POD 10 in functional New York Heart Association class I without any signs of angina pectoris. He received phenprocoumon with a target international normalized ratio (INR) of 2.0 to 2.5 and acetylsalicylic acid $(100 \mathrm{mg} / \mathrm{d})$. Seven weeks postoperatively, the patient
License terms

Stuttgart · New York

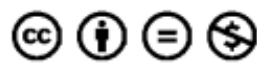

received

December 3, 2016

accepted

February 17, 2017
DOI http://dx.doi.org/

10.1055/s-0037-1601315. ISSN 2194-7635. 
was readmitted to our hospital due to new-onset angina pectoris Canadian Cardiovascular Society IV. The electrocardiogram at admission showed no abnormalities. Initial serum testing for troponin-I was negative $(0.02 \mu \mathrm{g} / \mathrm{L}$; upper reference limit $0.05 \mu \mathrm{g} / \mathrm{L}$ ) but became slightly elevated after 3 hours $(0.32 \mu \mathrm{g} / \mathrm{L})$. Transthoracic echocardiography confirmed regular function of the aortic valve prosthesis, and regional wall motion abnormalities were ruled out. Coronary angiography showed no new relevant stenoses in the native coronary system. However, a leakage of contrast medium from the LIMA-OM2 anastomosis into a heretofore indefinable spherical paraventricular/para-arterial space and thrombolysis in myocardial infarction (TIMI) 1 flow in the distal OM2 was documented. The reduced coronary flow in OM2 was attributed to impaired inflow due to anastomosis leakage ( $\mathbf{- F i g . 1}$ ). The etiology and anatomic architecture of this spherical mass was not distinguishable by angiography alone. Since a connection to the heart chambers was suspected, cardiac computed tomography (CT) was performed. CT showed a hematoma (measuring $5.5 \times 3.1 \times 5.0 \mathrm{~cm}$ ) connected to the LIMA-OM2 anastomosis and thereby compressing the OM2 by several centimeters. Any further connection to either cardiac cavities or the pericardium was excluded. The hematoma appeared to localize subepicardially and not intrapericardially (-Fig. 2).

As the patient suffered from angina pectoris and myocardial ischemia of the distal OM2 territory, the heart team decided to perform surgical re-exploration to remove the hematoma and restore the integrity of the anastomosis. During the course of the operation, we observed that the intrapericardial space was well cicatrized and free from any hematoma. As excessive scar tissue was present at this time point after the index operation and the localization of the hematoma required extensive mobilization of the heart, extracorporeal circulation was used. The subepicardial hematoma was observed to be without connection to the pericardial space ( $\boldsymbol{\sim}$ Fig. $\mathbf{3 A}$ ) at the lateral cardiac wall. After evacuation of the hematoma, bleeding from a small anastomosis suture leak was identified and closed with a single suture by adapting the adventitial tissue (Prolene 8/0) (-Fig. 3C, D). As we observed some diffuse bleeding from the preparation region around the anastomosis, a hemostatic patch (Veriset) was applied (-Fig. 3E). The epicardial layer that had contained the hematoma was left open. Transit-time flow measurement of the bypass revealed appropriate flow (19 $\mathrm{mL} / \mathrm{min})$.

Control coronary angiography performed on POD 4 showed a good function of the LIMA-OM2 bypass and TIMI 3 flow with a decompressed lumen of the OM2 (-Fig. 4). On POD 7, a pericardial effusion ( $400 \mathrm{~mL}$; exudative, hemoglobin $1.3 \mathrm{~g} / \mathrm{dL}$ ) was removed by ultrasound-guided pericardiocentesis and immunosuppressive therapy with prednisolone and ibuprofen for postcardiotomy syndrome was initiated. The patient recovered well subsequently and was discharged from the hospital on POD 15.

\section{Discussion}

To our knowledge, this is the first description of a spontaneous secondary subepicardial hematoma during the very
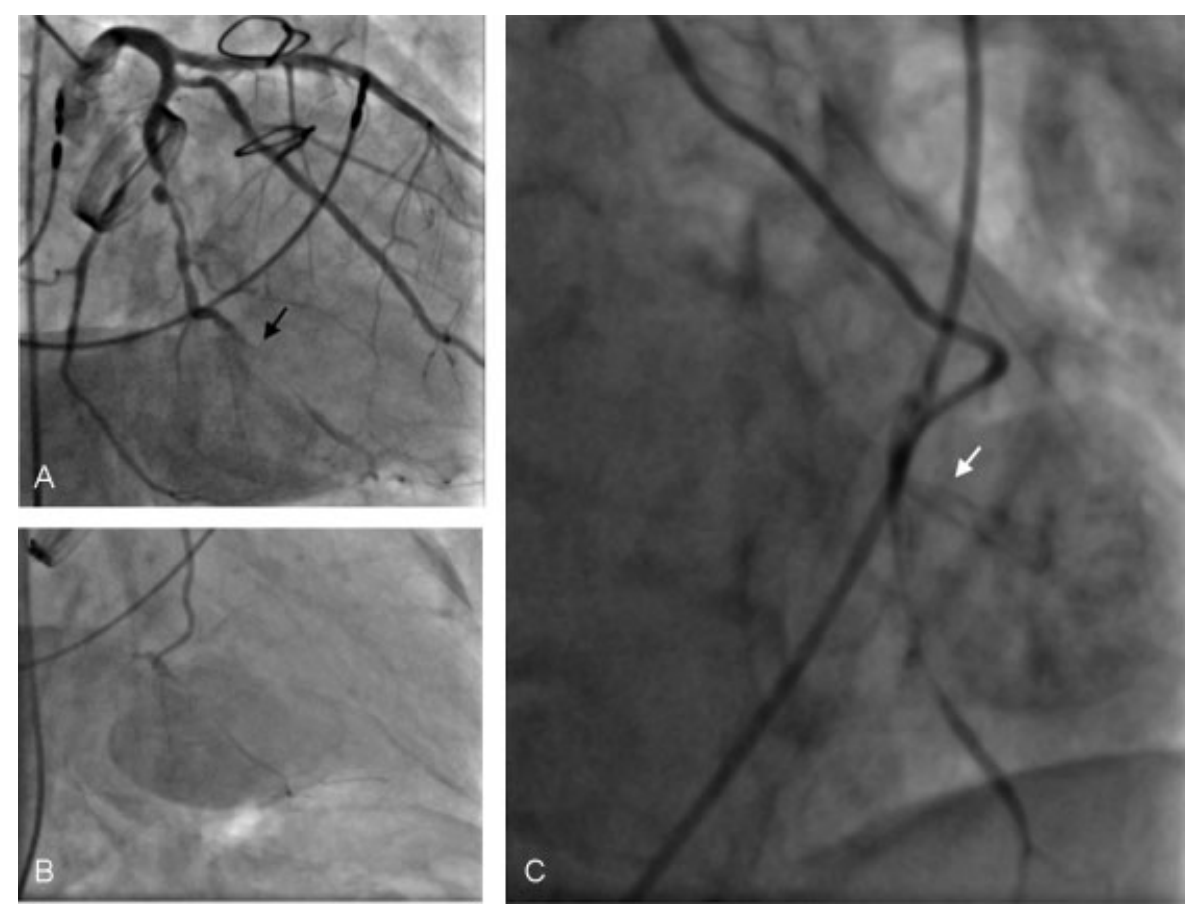

Fig. 1 Coronary angiogram (A) RAO view of native coronary vessels. The OM2 shows a long-segment discontinuity (black arrow) around the bypass anastomosis. (B) RAO view of the LIMA-OM2 bypass: from the anastomosis, there is retrograde flow into the OM2, TIMI 2 flow into the distal OM2, and a large spherical space is contrast enhanced. (C) LAO view of the LIMA-OM2 bypass anastomosis shows an apparent leak of contrast medium at the region of the anastomosis into a paraventricular spherical space (white arrow). LAO, left anterior oblique; LIMA, left internal mammary artery; OM2, obtuse marginal branch 2; RAO, right anterior oblique. 

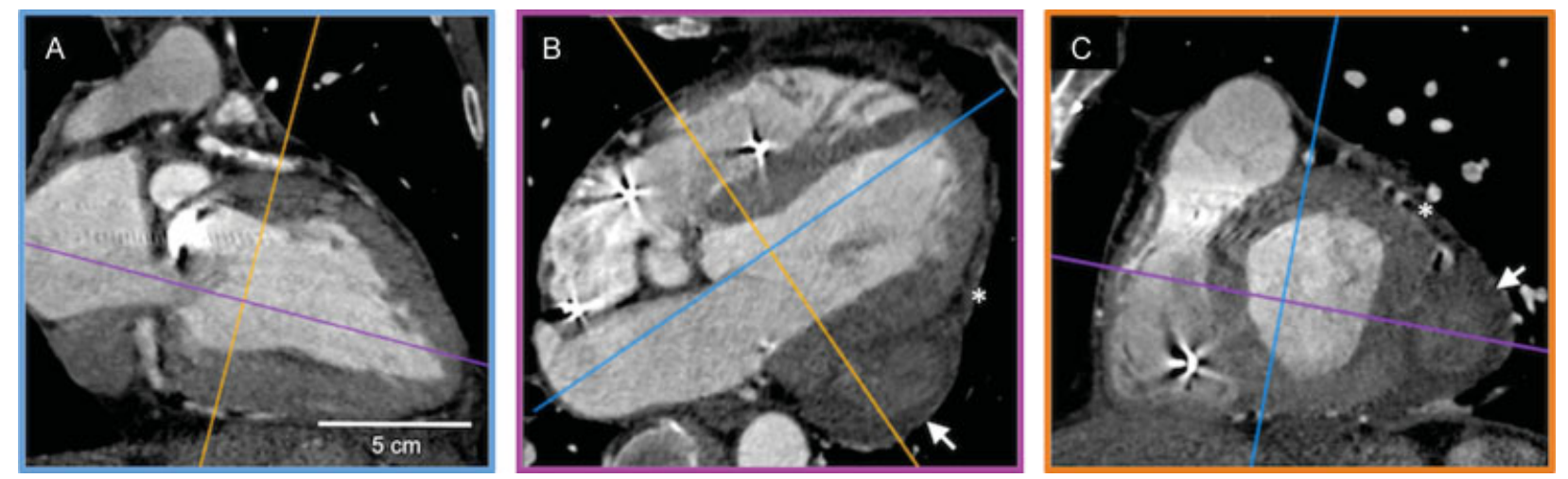

Fig. 2 Multiplanar reconstruction of the cardiac CT (ECG-triggered mid-diastolic recording). (A) Two-chamber view of the left ventricle and the left atrium. (B) Four-chamber view. (C) Short-axis view. Imaging shows a spherical space (white arrows) adjacent to the basal lateral wall of the left ventricle (diameter $5.5 \times 3.1 \times 5.0 \mathrm{~cm}$ ). There is slight contrast medium enhancement in this space. The space can be anatomically localized outside the myocardium and appears not to be connected to the pericardium (asterisks), thus being bordered by the epicardium. CT, computed tomography; ECG, electrocardiogram.

late postoperative period after CABG. We suspect bleeding to have started after primary healing of the anastomosis was completed, resulting in an entirely encapsulated hematoma within the epicardial layer. Previously published case reports referred to subepicardial hematomas after attempted coronary intervention in bypass grafts or anastomoses. ${ }^{4-6}$ Furthermore, usage of heart positioning devices for offpump CABG can induce epicardial trauma and hematomas. ${ }^{7}$
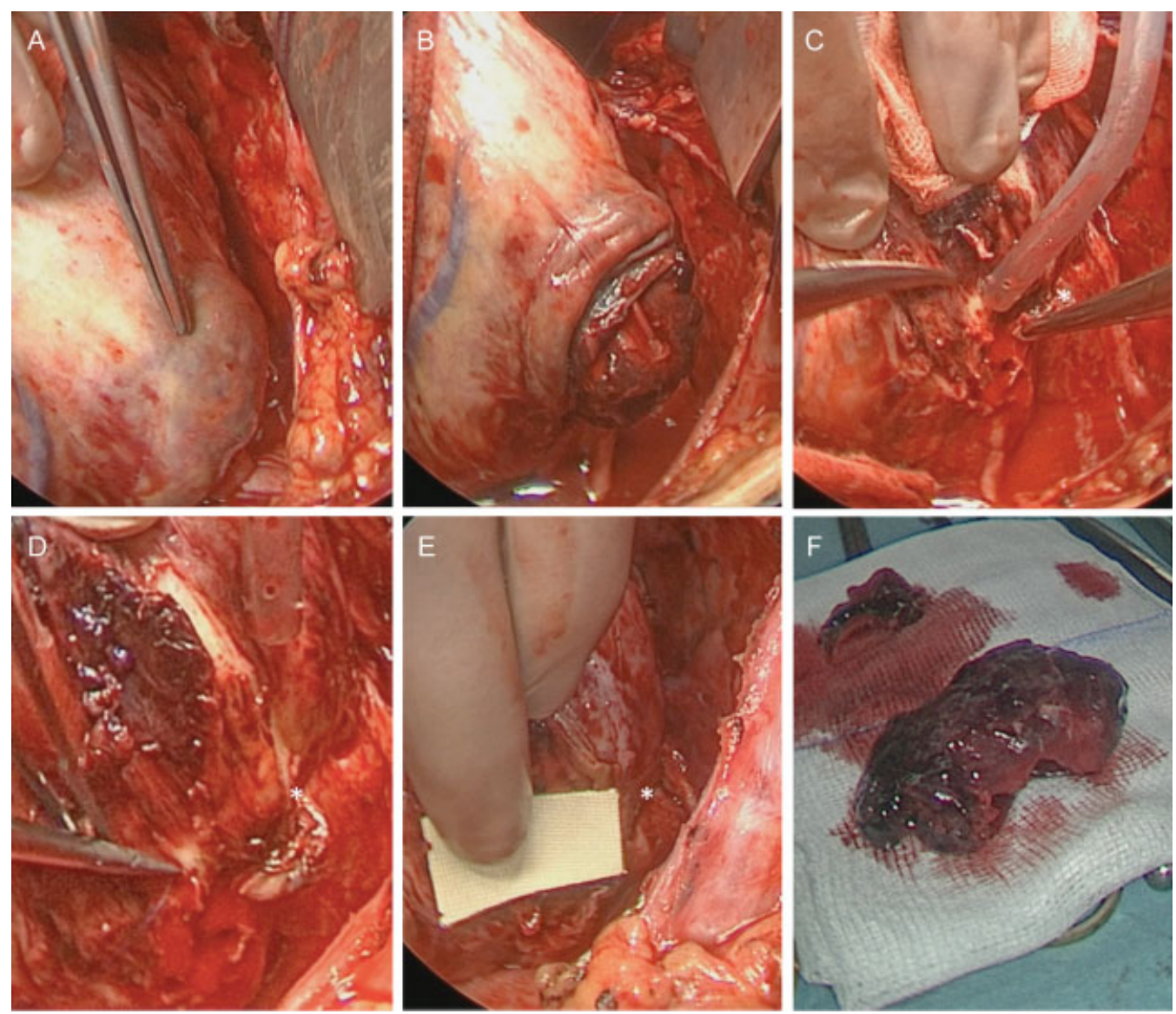

Fig. 3 Surgical removal of the subepicardial hematoma and repair of the LIMA-OM2 bypass anastomosis. View into the operation site from caudal-anterior perspective (course of the bypass in (c)-(e): white asterisks). (A) The patient is on cardiopulmonary bypass and the heart elevated to the right. The exposed hematoma on the left lateral wall is located subepicardially without connection to the pericardial space. (B) After opening the epicardial cover of the hematoma, a partially organized clot is removed. (C) Exposure of the anastomosis: a small defect with pulsating bleeding is identified. (D) The defect is repaired with a single suture. (E) To further secure the site of anastomosis, a hemostatic patch (Veriset) is applied. (F) Removed hematoma mass. LIMA, left internal mammary artery; OM2, obtuse marginal branch 2. 

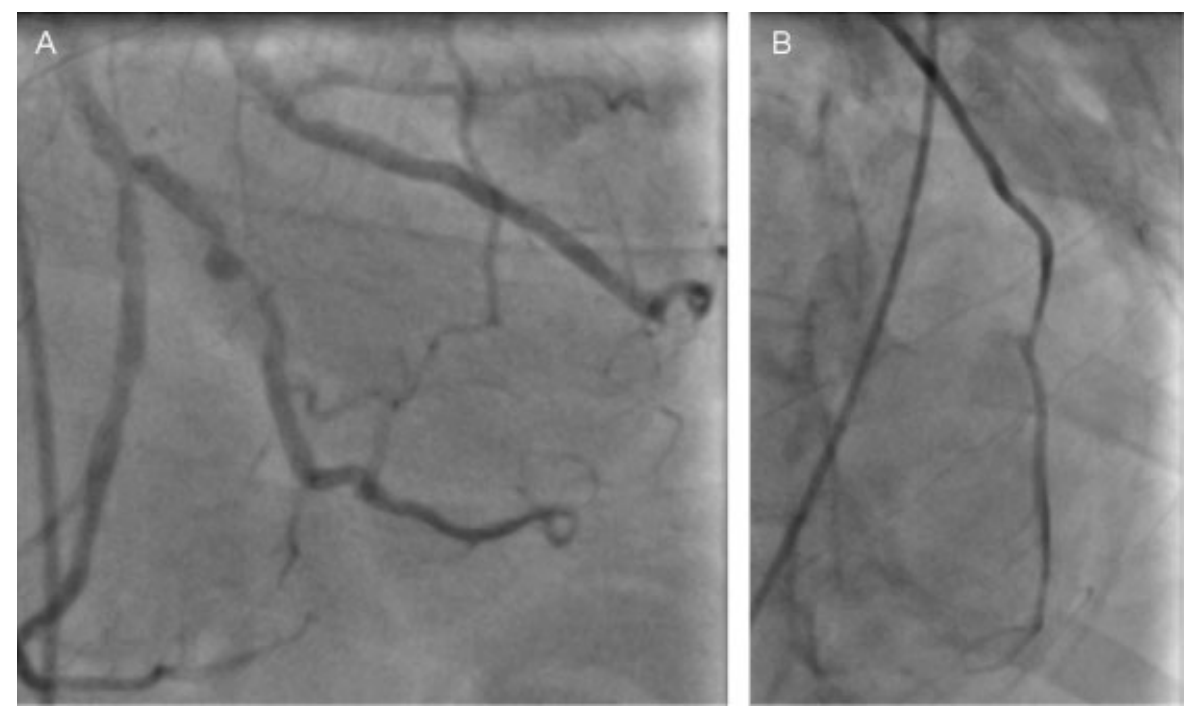

Fig. 4 Coronary angiogram on POD 4. (A) The native left circumflex artery shows the previously known stenosis of OM2. The OM2 distal to the bypass anastomosis shows no narrowing. (B) The LIMA-OM2 bypass shows good function without leakage of contrast medium and TIMI 3 flow in the distal OM2. LIMA, left internal mammary artery; OM2, obtuse marginal branch 2; POD, postoperative day; TIMI, thrombolysis in myocardial infarction classification.

Another potential pathomechanism includes trauma with sudden tension leading to dissection of the anastomosis. None of these effectors was relevant to the current case. As the patient was set on vitamin $\mathrm{K}$ antagonists and acetylsalicylic acid, potentially excessive anticoagulation could have induced ongoing bleeding. However, from the patients' records with INR controls twice weekly, no phases of hyperanticoagulation are evident. However, intermittent hyperanticoagulation cannot be excluded and bleeding is likely to be supported by the anticoagulation therapy.

The exact localization of the hematoma was not possible using coronary angiography alone, but CT provided excellent accuracy.

Due to persistent symptoms and myocardial ischemia, treatment options included interventional or surgical therapy. We considered treatment with percutaneous coronary intervention of the native OM2 and application of a polytetrafluoroethylene (PTFE)-covered coronary stent within the region of the LIMA-OM2 anastomosis. Several factors, however, spoke against using this method: first, the hematoma-induced compression of the distal OM2 would not have been resolved; second, the bleeding from the anastomosis would not definitively have been stopped; and third, a high risk of perforation of the anastomosis with consecutive severe bleeding would have been expected. In addition, long-term patency of PTFE-covered coronary stents might be inferior as restenosis rates of up to $21 \% 10$ months after treatment of coronary artery aneurysms have been reported. ${ }^{8}$ In the treatment of saphenous vein graft stenosis, the use of PTFE-covered stents compared with bare metal stents led to a higher rate of restenosis (68.3 vs. $51.8 \%$, $p=0.007$ ) after 5 years. ${ }^{9}$ Although the surgical approach is more invasive, the complete removal of the hematoma was achievable and relief of hematoma-induced compression of the distal coronary artery was provided. Furthermore, direct exploration and sophisticated repair of the anastomosis can be safely performed using a surgical approach with reasonable risk profiles. Due to the location of the hematoma and excessive pericardial adhesions, an on-pump approach was chosen during the reoperation. An off-pump strategy would have been considerable in situations with easier exposure of the hematoma.

In summary, we present for the first time a case of a rare complication after CABG in the late postoperative period. Efficient diagnostic and therapeutic management was achieved by a heart team approach involving interventional cardiologists, cardiac imaging specialists, and cardiac surgeons.

\section{References}

1 Shavadia J, Norris CM, Graham MM, Verma S, Ali I, Bainey KR. Symptomatic graft failure and impact on clinical outcome after coronary artery bypass grafting surgery: results from the Alberta Provincial Project for Outcome Assessment in Coronary Heart Disease registry. Am Heart J 2015;169(06):833-840

2 Dacey LJ, Munoz JJ, Baribeau YR, et al; Northern New England Cardiovascular Disease Study Group. Reexploration for hemorrhage following coronary artery bypass grafting: incidence and risk factors. Arch Surg 1998;133(04):442-447

3 Hosono M, Ueda M, Suehiro S, et al. Neointimal formation at the sites of anastomosis of the internal thoracic artery grafts after coronary artery bypass grafting in human subjects: an immunohistochemical analysis. J Thorac Cardiovasc Surg 2000;120(02): 319-328

4 Furushima H, Matsubara T, Tamura Y, et al. Coronary artery perforation with subepicardial hematoma. Cathet Cardiovasc Diagn 1997;41(01):59-61

5 Quan VH, Stone JR, Couper GS, Rogers C. Coronary artery perforation by cutting balloon resulting in dissecting subepicardial 
hematoma and avulsion of the vasculature. Catheter Cardiovasc Interv 2005;64(02):163-168

6 Holinski S, Konertz W, Beholz S. Covered perforated aortocoronary saphenous vein graft aneurysm. J Card Surg 2009;24(01): 91-93

7 Ariyama J, Imanishi H, Nakagawa H, Kitamura A, Hayashida M. Epicardial hematoma and myocardial ischemia following application of Starfish stabilizer: an uncommon complication of the device. J Anesth 2010;24(05):801-802
8 Szalat A, Durst R, Cohen A, Lotan C. Use of polytetrafluoroethylene-covered stent for treatment of coronary artery aneurysm. Catheter Cardiovasc Interv 2005;66(02):203-208

9 Stone GW, Goldberg S, O'Shaughnessy C, et al. 5-year follow-up of polytetrafluoroethylene-covered stents compared with bare-metal stents in aortocoronary saphenous vein grafts the randomized BARRICADE (barrier approach to restenosis: restrict intima to curtail adverse events) trial. JACC Cardiovasc Interv 2011;4(03): 300-309 